\title{
Omental Torsion in a Child - a Case Report
}

\author{
Baker Aㄹ Helal Uddin AKM², Karim MS³
}

\begin{abstract}
Omental torsion, a very rare cause of acute abdominal pain, mimicking acute appendicitis .A boy of 11 years, presented to us with the symptoms and signs suggestive of acute appendicitis clinically, was admitted through OPD of Apollo Hospitals. His laboratory reports were also in favor of clinical diagnosis. Laparoscopic appendicectomy was planned. But on laparoscopic intra-abdominal inspection it was found to be a case of Omental torsion, distal part was closely adherent to the ascending colon and blackish in color. So, procedure was converted into laparotomy and resection of the tensioned and gangrenous part of the omentum including appendicectomy was done. His post operative period was uneventful. Although torsion of the omentum is a rare clinical entity, but accurate pre-operative clinical assessment and relevant study including imaging and CT abdomen may reduce the diagnostic dilemma between omental torsion and acute appendicitis.
\end{abstract}

\section{Keywords}

Omental torsion, Laparoscopy, Laparotomy

\section{Case History}

An eleven years old boy was admitted into Apollo Hospitals Dhaka with the complaints of right lower abdominal pain for 3 days and fever for 1 day. He had also history of similar attack of pain few days back which was of short duration and self-limiting. His physical examination revealed tenderness all over the abdomen, maximum at the right iliac fossa and the right lumber area.

His laboratory findings was moderate leucocytes (16,500/cmm), CRP was 8.73 mg\%. Imaging study (done before coming to our hospitals) commented an inflammatory mass in the right iliac fossa, possibly appendicular lump. The patient was looking toxic at the time of examination and right side was very tender and could not move his right leg. The patient was considered as an urgent surgical case and a decision for laparoscopic evaluation with a view that in case of laparoscopic findings of appendicular lump, nothing much would be done. During the procedure, omentum was found twisted and discolored and distal part was adherent with the ascending colon and caecum, small amount of blood was also found in the right paracolic gutter, appendix was apparently healthy looking. Considering the intense adhesion of omentum, surrounding edema, an open approach was decided. So the procedure was converted into laparotomy. The twisted as well as gangrenous part of the omentum was resected, gently separated from ascending colon and appendicectomy was also done after consultation with the guardians to avoid future diagnostic confusion. Abdomen was toileted; a drain was kept in the pouch of Morrison through a separate wound. Then abdominal wounds were closed in layers accordingly. In the post operative period, the boy was doing well. His drain tube was removed on 3rd post-operative day. Check dressing was done on 5th post-operative day and the patient was discharged on the same day. Histopathological report was gangrenous omentum and acute appendicitis. We labeled it as case of primary right sided omental torsion. 


\section{Discussion}

Omental torsion is a rare pathology presenting as acute abdominal pain resembling the pain of acute appendicitis. Omental torsion in an adult, it was reported by Dr. A.K. Bhaya and A. Rahman. ${ }^{1}$ The patient was 35 years old presented with acute abdomen with co-existent inguinal hernia (omentocele). ${ }^{1}$ Primary omental torsion, extremely rare in children. A 10 years retrospective study among 2734 children operated for acute appendicitis, 5 were diagnosed with Primary omental torsion ${ }^{2}$ in a renowned hospital. Torsion occurs along the long axis of omentum. It can be primary or secondary. ${ }^{3}$ In primary omental torsion, it is always unipolar, distal pole is free and cause is unknown. In our case, was right sided primary omental torsion. There was no pathology detected in the distal part where it was attached to normal ascending colon by only flimsy adhesion. In secondary variety, distal part is attached to any intra-abdominal pathology, such as tumour, cyst and others, so it is bipolar. ${ }^{4}$ Primary omental torsion was first described by Eitel in 1899 and is very rare, with an incidence of $0.1 \%$ in children suffering from acute abdominal pain. ${ }^{5}$ However, whatever may be the type, either primary or secondary, it is very difficult to diagnose pre-operatively. But surgeons should always consider it in the differential diagnosis of acute abdomen which includes acute appendicitis, torsion of the ovarian cyst (in female), acute cholecystitis, diverticulitis and others. ${ }^{6}$ Unfortunately, the symptoms and signs do not present in any characteristic pattern that suggests the diagnosis in favor of omental torsion. 6,7

Regarding our case, clinically and laboratory findings were in favor to diagnose the boy as a case of acute appendicitis. Although imaging comment was very close to the appendicular lump, patient was toxic and suffering from intense abdominal pain. Usually the clinical symptoms and laboratory findings are similar to acute appendicitis. ${ }^{2}$ In our case, patient presented with right lower abdominal pain with high CBC and CRP. We arranged for laparoscopic evaluation and possible appendectomy. But the procedure became diagnostic only, conversion into laparotomy was needed. In modern practice, the most of the surgeons add laparoscopy as diagnostic tool to confirm intra-abdominal pathology in addition to imaging and CT evaluation. ${ }^{8}$

In case of acute appendicitis, the ultimate management is appendicectomy, either by laparoscopy or by laparotomy. But surgery is not needed in every cases of Omental torsion. Some cases may be managed conservatively without any residual effect.8,9 CT scan may be helpful in the pre-operative diagnosis in some cases and surgeon may take accurate decision for proper management of the individual case. ${ }^{6,7}$

\section{Conclusion}

Omental torsion is a rare cause of acute abdomen but should be included in the differential diagnosis of acute abdomen. CT scan of abdomen may help in pre-operative diagnosis but laparoscopy is the ultimate step both for accurate diagnosis and for therapeutic purpose. In this way, unnecessary appendicectomy can be avoided.

In our case the histopathology showed the patient was also suffering from acute appendicitis. 


\section{References}

1. A. K. Bhaya, A. Rahman. Omental torsion: rare entity in common scenario diagnosed prospectively on CT scan. PULSE Medicine Journal of Apollo hospitals Dhaka. Dec 2006. 1(1).

2. Mavridis G, Livaditi E, Baltogiannis N, Vasiliadou E, Christopoulos-Geroulanos G. Primary omental torsion in children: ten-year experience. Pediatr Surg Int. 2007 Sept;23(9):879-82.

3. Sable S, Gandhi V, Nagral S and Nagral A. Secondary omental torsion. Jaslok Hospital, Mumbai, India. BMJ Case Report. 2012; doi: 10.1136/bcr. 12.2011.5447

4. Jain P, Chhabra S, Parikh K and Vaidya A. Omental torsion. Journal of Indian Association of Pediatric Surgeons. 2008;13(4):151-52.

5. Sencan A, Arslan O, Yilmaz O, Ayhan S and Mir E. A rare cause of acute abdomi nal pain: primary torsion of omentum majus. Celal Bayar University,
Department of Pediatric Surgery, Manisa.

6. Stefano S, Edoardo R, Andrea M, Emanuele R, Chiara L, Renato S, Luisito DM and Valter F. Primay Omental torsion: a case report. PMC

7. Nina B and Paul S. A diagnostic challenge: primary omental torsion and literature review-a case report. World Journal of Emergency Surgery. 2009;4:40 doi: 10. 1186/1749-7922-4-40. Online http://www.wies.org/content/4/1/40

8. Syed DA and Syed SR. Common presentation, uncommon diagnosis, primary torsion. Liakat National Hospital and Medical College, Karachi

9. Khattala K, Tenrkarang SS, Elmadi A Rami M and Boubdallah Y. Primary Omental torsion: case report. Department of Paediatric Surgery, University Hospital Hassan II, Fez, Morocco. 